\title{
55. Potent Novel Mutagens Produced by Broiling Fish under Normal Conditions
}

\author{
By Hiroshi KasaI, *) Ziro YAmaIzUmi,*) Keiji WaKabayashi, **) \\ Minako NaGAo,**) Takashi SUGIMURA,**) \\ Sigeyuki YoKoyama,***) Tatsuo MrYazaWA,***) \\ Neil E. SPINGarn,****) John H. WeISBURGER,****) \\ and Susumu NishimuRA*) \\ (Communicated by Hamao UmezawA, M. J. A., May 12, 1980)
}

Introduction. Mutagenicity has been demonstrated in smoke condensates from broiling fish and beefsteak and in the charred surface of fish and beefsteak (Nagao et al., 1977; Sugimura et al., 1977; Rappaport et al., 1979). Further studies showed that pyrolysis products of proteins and amino acids contained strong mutagens (Sugimura and Nagao, 1979a; Matsumoto et al., 1977), and active compounds were isolated from pyrolysates of various amino acids (Sugimura et al., 1977; Yamamoto et al., 1978; Wakabayashi et al., 1978; Kosuge et al., 1978) and proteins (Kosuge et al., 1978; Yoshida et al., 1978). Among these compounds, 3-amino-1,4-dimethyl-5Hpyrido[4,3-b] indole (Trp-P-1) and 3-amino-1-methyl-5H-pyrido[4,3$b$ ]indole (Trp-P-2) from tryptophan (Sugimura et al., 1977), and 2-amino-6-methyldipyrido[1,2-a: $\left.3^{\prime}, 2^{\prime}-d\right]$ imidazole (Glu-P-1) and 2aminodipyrido[1,2- $\left.a: 3^{\prime}, 2^{\prime}-d\right]$ imidazole (Glu-P-2) from glutamic acid (Yamamoto et al., 1978) are very strong mutagens, especially towards Salmonella typhimurium TA98 with metabolic activation. The in vitro (Takayama et al., 1977; 1978) and in vivo (Ishikawa et al., 1979; unpublished data of our laboratory) carcinogenicities of Trp-P-1 and Trp-P-2 were already demonstrated.

For evaluation of the effects of mutagens in cooked foods on humans, it is important to determine the properties of mutagenic substances that are actually present in foods. Mutagenicity has been demonstrated in fried hamburgers (Commoner et al., 1978; Pariza et al., 1979; Spingarn and Weisburger, 1979) and fried potatoes (Spin-

*) Biology Division, National Cancer Center Research Institute, Chuo-ku, Tokyo 104.

**) Biochemistry Division, National Cancer Center Research Institute, Chuo$\mathrm{ku}$, Tokyo 104.

***) Department of Biophysics and Biochemistry, Faculty of Sciences, University of Tokyo, Bunkyo-ku, Tokyo 113, Japan.

****) Naylor Dana Institute for Disease Prevention, American Health Foundation, Valhalla, NY 10595, USA. 
garn et al., 1980b), but the compounds responsible for this activity have not yet been isolated and identified. We previously detected TrpP-1 and Trp-P-2 in broiled sardine, but these compounds accounted for only $1.5 \%$ of the total mutagenicity present (Yamaizumi et al, 1980 ). It strongly suggests that most of the mutagenicity in broiled sardine is due to compounds other than the series of newly described heterocyclic amines, and in fact we found that the basic fraction of broiled sardine contained an unidentified mutagenic substances (Kasai et al., 1979).

This communication describes two new potent mutagens isolated from the neutral fraction of broiled sardines. They are novel mutagens with a six-six-five membered ring system and their structures are 2-amino-3-methylimidazo[4,5- $d]$ quinoline (IQ) and 2-amino-3,5dimethylimidazo [4,5- $d$ ] quinoline (methyl-IQ). This is a first report of elucidation of the structures of new mutagens isolated from ordinarily cooked food.

Isolation of pure mutagens from the neutral fraction of broiled sardines. A sample of $3 \mathrm{~kg}$ of sun-dried sardines (named "Maruboshi" in Japanese) were broiled in the ordinary way as described previously (Kasai et al., 1979). Then they were ground up and extracted with methanol. The extract was evaporated and the residue, which showed strong mutagenicity to S. typhimurium TA98 in the presence of S9 mix, was further fractionated as described previously (Kasai et al., 1979). The neutral fraction thus obtained, which contained $40 \%$ of the original mutagenicity, was subjected successively, to Diaion HP-20 column chromatography, chloroform-methanol-water partitioning, and Sephadex LH-20 column chromatography. The active fraction was then chromatographed on a column of silica gel with $10 \%$ methanol in chloroform as solvent. The mutagens were roughly separated into two components (compound 1 and compound 2) by this procedure. Final purification of the two components was achieved by successive application of high pressure liquid chromatographies as described in Fig. 1. Approximately $0.06 \mathrm{mg}$ of each purified compound was obtained from $3 \mathrm{~kg}$ of sardines. Actually $200 \mu \mathrm{g}$ of each material, obtained from $10 \mathrm{~kg}$ of sardines, was used for structural studies. Table I shows the increase in specific activity at each step of purification. The final purified samples of compounds 1 and 2 showed high mutagenic activities to TA98 strain of Salmonella of 8,000 and 18,500 revertants $/ \mu \mathrm{g}$, respectively.

Elucidation of the structures of the mutagens. The structure of compound 1 was proposed as I based mainly on its $270 \mathrm{MHz}{ }^{1} \mathrm{H}-\mathrm{NMR}$ spectrum, and low- and high-resolution mass spectra. The mass spectrum of compound 1 showed a high intensity molecular ion $\left(\mathrm{M}^{+}\right)$ 

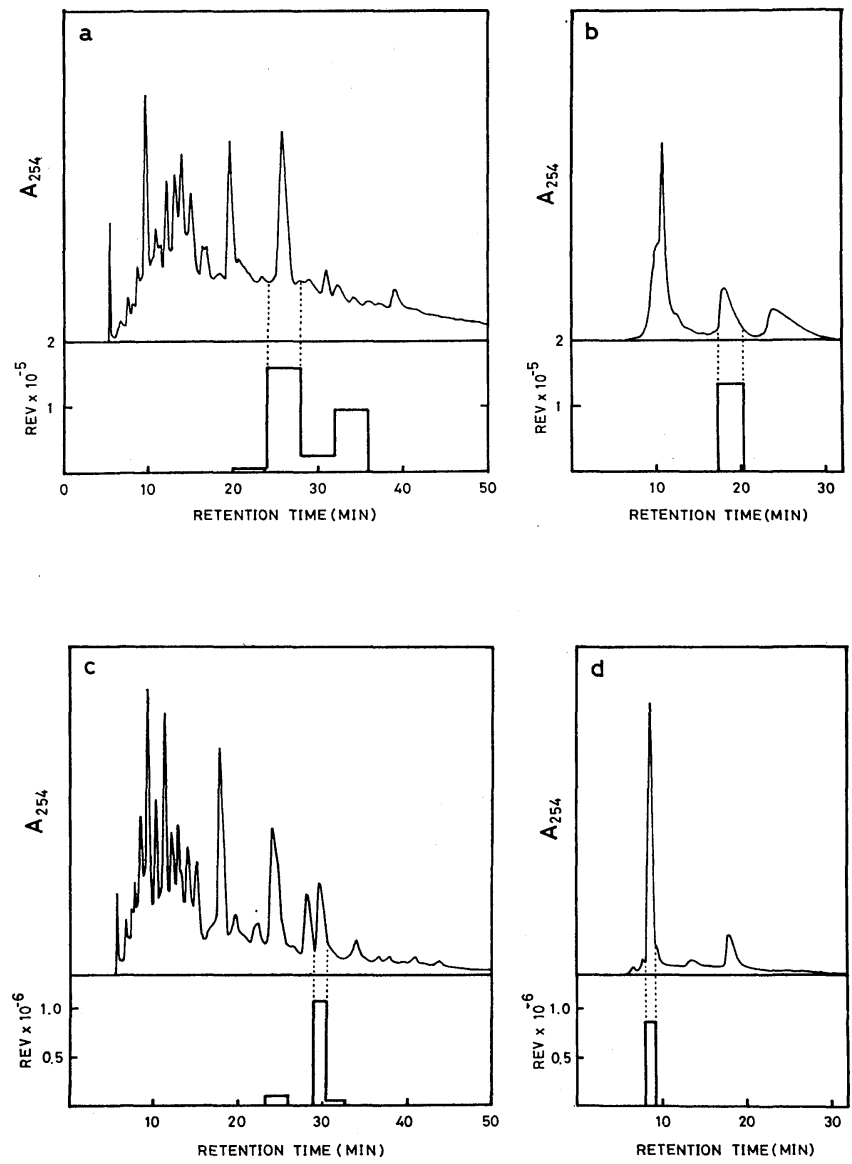

Fig. 1. Purification of the mutagens by high pressure liquid chromatography (HPLC). (a) The mutagenic fractions eluted later (Fr. II, $1.6 \mathrm{mg}$ ) from silica gel were dissolved in $0.2 \mathrm{ml}$ of $40 \%$ methanol and injected. Conditions for HPLC were as follows: Instrument, Shimazu LC-3A; column $\mu$ Bondapak $\mathrm{C}_{18}$ (Waters $7.9 \times 300 \mathrm{~mm}$ ); flow rate, $2.0 \mathrm{ml} / \mathrm{min}$; eluent, $40 \%-70 \%$ methanol, linear gradient. The profiles of $A_{254}$ (upper figure) and mutagenic activity (revertants/plate/min, lower figure) are shown. The second minor peak of activity was that of compound 2. (b) The mutagenic fraction obtained by HPLC-a (fraction between dotted lines, $0.13 \mathrm{mg}$ ) was dissolved in $0.2 \mathrm{ml}$ of $60 \%$ methanol and injected. Column, $\mu$ Bondapak $\mathrm{C}_{18}$ (Waters $4 \times 300 \mathrm{~mm}$, two columns connected in series); flow rate, $1.0 \mathrm{ml}$ / min; eluent, $60 \%$ methanol. (c) The mutagenic fractions eluted earlier (Fr. I, $3 \mathrm{mg}$ ) from silica gel were injected. Conditions were as for (a). (d) The mutagenic fraction $(0.18 \mathrm{mg})$ obtained by HPLC-c was injected. Conditions were as for (b) except that the solvent system was $40 \%$ methanol, $0.04 \%$ formic acid. 
Table I. Purification of compounds 1 and 2 from broiled sardines

\begin{tabular}{lccc}
\hline \multicolumn{1}{c}{ Purification steps } & $\begin{array}{c}\text { Weight } \\
(\mathrm{mg})\end{array}$ & $\begin{array}{c}\text { Specific activity } \\
(\text { revertants } / \mathrm{mp})\end{array}$ & $\begin{array}{c}\text { Total activity } \\
\left(\text { revertants } \times 10^{-4}\right)\end{array}$ \\
\hline $\mathrm{MeOH}$ extract & 817,000 & 289 & 23,600 \\
$\mathrm{Et}_{2} \mathrm{O}-\mathrm{H}_{2} \mathrm{O}$ partition & 537,000 & 178 & 9,560 \\
$\mathrm{HP}_{2} \mathrm{O}$ & 54,000 & 974 & 5,260 \\
$\mathrm{CHCl}_{3}-\mathrm{MeOH}-\mathrm{H}_{2} \mathrm{O}$ partition & 16,600 & 3,520 & 5,840 \\
$\mathrm{LH}-2 \mathrm{O}$ & 443 & 84,400 & 3,740 \\
$\mathrm{LH}-2 \mathrm{O}$ (rechromato.) & 89.4 & 243,000 & 2,170 \\
Silica Gel-Fr. I & 3.0 & 622,000 & 187 \\
HPLC-c & 0.18 & $9,720,000$ & 175 \\
HPLC-d, compound 2 & 0.06 & $18,500,000$ & 103 \\
Silica Gel-Fr. II & 1.6 & 549,000 & 88 \\
HPLC-a & 0.13 & $4,920,000$ & 64 \\
HPLC-b, compound 1 & 0.06 & $8,000,000$ & 50 \\
\hline
\end{tabular}

at $\mathrm{m} / \mathrm{e} 198$ and fragment ion at $\mathrm{m} / \mathrm{e} 183\left(\mathrm{M}^{+}-15\right)$, suggesting that compound 1 has a stable heterocyclic nucleus and methyl group. The elemental composition of compound 1 was determined to be $\mathrm{C}_{11} \mathrm{H}_{10} \mathrm{~N}_{4}$ by exact mass measurement (calculated mass, 198.0906; observed mass, 198.0900). The $270 \mathrm{MHz}{ }^{1} \mathrm{H}-\mathrm{NMR}$ spectrum of compound 1 measured in $\mathrm{CDCl}_{3}$ showed five aromatic protons, methyl protons and broad amino protons. The chemical shifts (ppm) and coupling constants $(\mathrm{Hz})$ are shown in Fig. 2. Three protons in the aromatic region

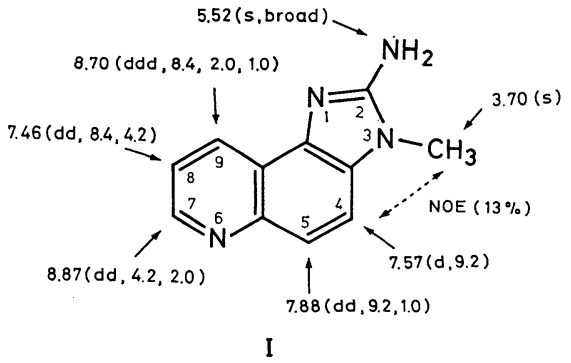

Fig. 2. $270 \mathrm{MHz}{ }^{1} \mathrm{H}-\mathrm{NMR}$ data for compound 1 in $\mathrm{CDCl}_{3}$. Chemical shifts (ppm) and coupling constants $(\mathrm{Hz})$ were shown by arrows.

(8.87, 8.70 and $7.46 \mathrm{ppm}$ ) were assigned to three protons, H-7, H-9 and $\mathrm{H}-8$, respectively, based on their chemical shifts and coupling constants. Another set of protons at 7.88 and $7.57 \mathrm{ppm}$, which were coupled to each other $(\mathrm{J}=9.2 \mathrm{~Hz})$, can be assigned to $\mathrm{H}-4$ and H-5. Long-range coupling $(\mathrm{J}=1 \mathrm{~Hz})$ was observed between $\mathrm{H}-5$ and $\mathrm{H}-9$. A methyl signal found at a considerably low field $(3.70 \mathrm{ppm})$ can be assigned to the N-methyl group. NOE was observed only for H-4 $(13 \%)$ when methyl protons were irradiated. The broad signal at $5.52 \mathrm{ppm}$ was assigned to protons of the amino group because this signal disappeared by the saturation transfer from the proton resonance of water in sample solution. The presence of a free amino group was confirmed by the mass spectral analysis of the trimethylsilyl deri- 
vative $\left(\mathrm{M}^{+}: 270\right)$.

The structure of compound 2 was proposed as II by comparing the $270 \mathrm{MHz}{ }^{1} \mathrm{H}-\mathrm{NMR}$ and high resolution mass spectrum data for compound 2 with those for compound 1. The mass-spectrum of compound 2 shows a molecular ion at $\mathrm{m} / \mathrm{e} 212$ and fragment ion at $\mathrm{m} / \mathrm{e}$ $197\left(\mathrm{M}^{+}-15\right)$. Exact mass measurement of compound 2 gave the elemental composition $\mathrm{C}_{12} \mathrm{H}_{12} \mathrm{~N}_{4}$ (calculated mass, 212.1063; observed mass, 212.1046). From these results it was concluded that an extra methyl group is present in compound 2. The presence of an extra methyl group in compound 2 is also supported by the fact that the UV spectra of compound 1 and compound 2 are similar except that the $\lambda \max$ of the former shows a blue shift of $2 \mathrm{~nm}$ relative to that of the latter ( $\lambda \max$ of compound 1 in methanol, $264 \mathrm{~nm}$; $\lambda \max$ of compound 2 in methanol, $266 \mathrm{~nm}$ ). The location of the extra methyl group was determined by comparing the ${ }^{1} \mathrm{H}-\mathrm{NMR}$ spectrum of compound 2 with that of compound 1 (Fig. 3 ). In the spectrum of compound 2 , there was

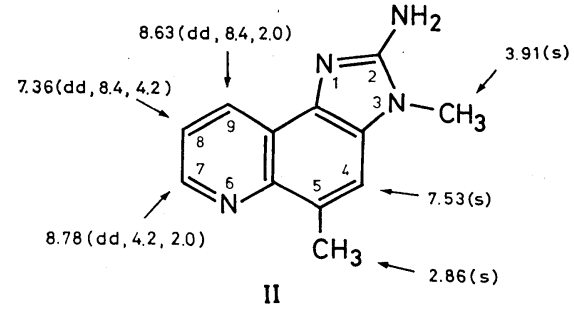

Fig. 3. $270 \mathrm{MHz}{ }^{1} \mathrm{H}-\mathrm{NMR}$ data for compound 2 in $\mathrm{CDCl}_{3}$. Chemical shifts (ppm) and coupling constants $(\mathrm{Hz})$ were shown by arrows.

no H-5 signal, the H-4 signal appeared as a singlet and an extra methyl signal appeared at $2.86 \mathrm{ppm}$. From these results we concluded that compound 2 has structure II, in which the extra methyl group is attached at position 5 .

Discussion. Two mutagens present in the neutral fraction of broiled sardines were concluded to be 2-amino-3-methylimidazo [4,5- $d]$ quinoline and 2-amino-3,5-dimethylimidazo[4,5-d] quinoline. They are hereafter abbreviated as IQ and methyl-IQ, respectively. They should be new mutagens which are commonly present in ordinary cooked foods. It is interesting that a mutagen recently isolated from heated beef extract seems to be identical to IQ, judging from its mass-, NMRand UV-spectra and chromatographic behavior (Spingarn et al., 1980a). Thus it is likely that IQ is widely distributed in a variety of broiled foods. It is possible that the mutagenic component previously detected in the basic fraction of broiled sardines (Kasai et al., 1979) is the same compound as IQ or methyl-IQ, because it behaves similarly as IQ or methyl-IQ in HPLC (Kasai et al., 1979), and IQ from beef extract was isolated from the basic fraction (Spingarn et al., 1980b). 
IQ and methyl-IQ have a six-six-five membered ring system, and they are the first mutagens of this type. It seems likely that in ultimate active forms in cells the amino group of IQ and methyl-IQ is converted to hydroxylamino group, as in the case of Trp-P-1 and Trp-P-2 (Sugimura and Nagao, 1979b), because S-9 mixture treatment is required for their mutagenic action.

Acknowledgement. The authors wish to thank Dr. Toshio Goto of Nagoya University for his valuable suggestion and criticism. This work was supported in part by funds from the Ministry of Health and Welfare, and the Ministry of Education, Science and Culture (H.K., M.N., T.S. and S.N.), the US National Cancer Institute's US-Japan Cooperative Cancer Program and Fellowship CA-06357 (N.E.S.), and USPHS grant CA-24217 (J.H.W.).

\section{References}

Commoner, B. et al. (1978) : Science, 201, 913-916.

Ishikawa, T. et al. (1979): Naturally Occurring Carcinogens-mutagens and Modulators of Carcinogenesis (eds. E. C. Miller et al.). Univ. Park Press, Baltimore, pp. 159-167.

Kasai, H. et al. (1979) : Cancer Lett., 7, 343-348.

Kosuge, T. et al. (1978) : Chem. Pharm. Bull., 26, 611-619.

Matsumoto, T. et al. (1977) : Mutat. Res., 48, 279-286.

Nagao, M. et al. (1977) : Cancer Lett., 2, 221-226.

Pariza, M. W. et al. (1979) : Cancer Lett., 7, 63-69.

Rappaport, S. M., McCartney, M. C., and Wei, E. T. (1979) : Cancer Lett., 8, 139-145.

Spingarn, N. E. et al. (1980a) : Cancer Letters, 9, 177-183.

Spingarn, N. E., Slocum, L. A., and Weisburger, J. H. (1980b) : Cancer Lett., 9, 7-12.

Spingarn, N. E., and Weisburger, J. H. (1979) : Cancer Lett., 7, 259-264.

Sugimura, T. et al. (1977) : Proc. Japan Acad., 53, 58-61.

Sugimura, T., and Nagao, M. (1979a) : CRC Critic. Rev. Toxicol., 6, 189-209.

Sugimura, T., and Nagao, M. (1979b) : Chemical Mutagens 6 (eds. Hollaender, A., and de Serres, F. J.). Plenum Press, New York, pp. 41-60.

Sugimura, T. et al. (1977) : Origin of Human Cancer (eds. Hiatt et al.). Cold Spring Harbor Lab., Cold Spring Harbor, pp. 1561-1577.

Takayama, S., Hirakawa, T., and Sugimura, T. (1978) : Proc. Japan Acad., 54B, 418-422.

Wakabayashi, K. et al. (1978) : Proc. Japan Acad., 54B, 569-571.

Takayama, S. et al. (1977) : Proc. Japan Acad., 53B, 126-129.

Yamaizumi, Z. et al. (1980) : Cancer Lett., 9, 75-83.

Yamamoto, T. et al. (1978) : Proc. Japan Acad., 54B, 248-250.

Yoshida, D. et al. (1978) : Biochem. Biophys. Res. Commun., 83, 915-920. 